POS PROCEEDINGS

\title{
The Tibet AS+MD Project; status report 2017
}

M. Amenomori, ${ }^{a}$ X. J. Bi, ${ }^{b}$ D. Chen, ${ }^{c}$ T. L. Chen, ${ }^{d}$ W. Y. Chen,,${ }^{b}$ S. W. Cui, ${ }^{e}$ Danzengluobu, ${ }^{d}$ L. K. Ding, ${ }^{b}$ C. F. Feng, ${ }^{f}{ }^{2}$ haoyang Feng, ${ }^{b}$ Z. Y. Feng, ${ }^{g}$ Q. B. Gou, ${ }^{b}$ Y. Q. Guo, ${ }^{b}$ H. H. He, ${ }^{b}$ Z. T. He, ${ }^{e}$ K. Hibino, ${ }^{h}$ N. Hotta, ${ }^{i}$ Haibing Hu, ${ }^{d}$ H. B. Hu, ${ }^{b}$ J. Huang, ${ }^{b}$ H. Y. Jia, ${ }^{g}$ L. Jiang, ${ }^{b}$ F. Kajino, ${ }^{j}$ K. Kasahara, ${ }^{k}$ Y. Katayose, ${ }^{l}$ C. Kato, ${ }^{m}$ K. Kawata, ${ }^{n}$ M. Kozai, ${ }^{o m}$ Labaciren, ${ }^{d}$ G. M. Le, ${ }^{p}$ A. F. Li, ${ }^{q f b}$ H. J. Li, ${ }^{d}$ W. J. Li, ${ }^{b g}$ C. Liu, ${ }^{b}$ J. S. Liu, ${ }^{b}$ M. Y. Liu, ${ }^{d}$ H. Lu, ${ }^{b}$ X. R. Meng, ${ }^{d}$ T. Miyazaki, ${ }^{m}$ K. Mizutani, ${ }^{k r}$ K. Munakata, ${ }^{m}$ T. Nakajima, ${ }^{m}$ Y. Nakamura, ${ }^{m}$ H. Nanjo, ${ }^{a}$ M. Nishizawa, ${ }^{s}$ T. Niwa, ${ }^{m}$ M. Ohnishi, ${ }^{n}$ I. Ohta, ${ }^{t}$ S. Ozawa, ${ }^{k}$ X. L. Qian, ${ }^{f b}$ X. B. Qu, ${ }^{u}$ T. Saito, ${ }^{v}$ T. Y. Saito, ${ }^{w}$ M. Sakata, ${ }^{j}$ T. K. Sako, ${ }^{x n}$ J. Shao, ${ }^{b f}$ M. Shibata, ${ }^{l}$ A. Shiomi, ${ }^{y}$ T. Shirai, ${ }^{h}$ H. Sugimoto, ${ }^{z}$ M. Takita ${ }^{*}{ }^{n}$ Y. H. Tan, ${ }^{b}$ N. Tateyama, ${ }^{h}$ S. Torii, ${ }^{k}$ H. Tsuchiya,${ }^{A}$ S. Udo, ${ }^{h}$ H. Wang, ${ }^{b}$ H. R. Wu, ${ }^{b}$ L. Xue, ${ }^{f}$ Y. Yamamoto, ${ }^{j}$ K. Yamauchi, ${ }^{l}$ Z. Yang, ${ }^{b}$ A. F. Yuan, ${ }^{d}$ T. Yuda ${ }^{\dagger n}$ L. M. Zhai, ${ }^{c}$ H. M. Zhang, ${ }^{b}$ J. L. Zhang, ${ }^{b}$ X. Y. Zhang, ${ }^{f}$ Y. Zhang, ${ }^{b}$ Yi Zhang, ${ }^{b}$ Ying Zhang, ${ }^{b}$ Zhaxisangzhu $^{d}$ and X. X. Zhou ${ }^{g}$ (The Tibet AS $\gamma$ Collaboration) 
${ }^{a}$ Department of Physics, Hirosaki University, Japan

${ }^{b}$ Key Laboratory of Particle Astrophysics, Institute of High Energy Physics, Chinese Academy of Sciences, China

${ }^{c}$ National Astronomical Observatories, Chinese Academy of Sciences, China

${ }^{d}$ Department of Mathematics and Physics, Tibet University, China

${ }^{e}$ Department of Physics, Hebei Normal University, China

${ }^{f}$ Department of Physics, Shandong University, China

${ }^{g}$ Institute of Modern Physics, SouthWest Jiaotong University, China

${ }^{h}$ Faculty of Engineering, Kanagawa University, Japan

${ }^{i}$ Faculty of Education, Utsunomiya University, Japan

${ }^{j}$ Department of Physics, Konan University, Japan

${ }^{k}$ Research Institute for Science and Engineering, Waseda University, Japan

${ }^{l}$ Faculty of Engineering, Yokohama National University, Japan

${ }^{m}$ Department of Physics, Shinshu University, Japan

${ }^{n}$ Institute for Cosmic Ray Research, The University of Tokyo, Japan

${ }^{o}$ Institute of Space and Astronautical Science, Japan Aerospace Exploration Agency (ISAS/JAXA),

Japan

${ }^{p}$ National Center for Space Weather, China Meteorological Administration, China

${ }^{q}$ School of Information Science and Engineering, Shandong Agriculture University, China

${ }^{r}$ Saitama University, Japan

${ }^{s}$ National Institute of Informatics, Japan

${ }^{t}$ Sakushin Gakuin University, Japan

${ }^{u}$ College of Science, China University of Petroleum, China

${ }^{v}$ Tokyo Metropolitan College of Industrial Technology, Japan

${ }^{w}$ Max-Planck-Institut für Physik, Deutschland

${ }^{x}$ Escuela de Ciencias Físicas y Nanotechnología, Yachay Tech, Ecuador

${ }^{y}$ College of Industrial Technology, Nihon University, Japan

${ }^{z}$ Shonan Institute of Technology, Japan

${ }^{A}$ Japan Atomic Energy Agency, Japan

E-mail: takitadicrr.u-tokyo.ac.jp

We built a large (approximately 4,000 $\mathrm{m}^{2}$ ) water Cherenkov-type muon detector array under the existing Tibet air shower array at 4,300 $\mathrm{m}$ above sea level, to observe 10-1000 TeV gamma rays from cosmic-ray accelerators in our Galaxy with wide field of view at very low background level. A gamma-ray induced air shower has significantly less muons compared with a cosmic-ray induced one. Therefore, we can effectively discriminate between primary gamma rays and cosmicray background events by means of counting number of muons in an air shower event by the muon detector array. We make a status report on the experiment.

35th International Cosmic Ray Conference - ICRC2017

10-20 July, 2017

Bexco, Busan, Korea

\footnotetext{
${ }^{*}$ Speaker.

${ }^{\dagger}$ Deceased.
} 


\section{Introduction}

The Tibet air shower array (Tibet AS: $\left.36,900 \mathrm{~m}^{2}\right)$ has been in operation at Yangbajing $(4,300 \mathrm{~m}$ above sea level) Tibet, China as a joint experiment between Japan and China. In contrast with narrow field-of-view imaging air Cherenkov telescopes (IACTs) which can make observation only in clear and moonless nights, it has continuously made a wide field-of-view (approximately 2 steradian) observation of cosmic rays and gamma rays in the northern sky. We have studied celestial gamma-ray sources in the $1 \mathrm{TeV}$ energy region and the chemical component of galactic primary cosmic rays in the knee $\left(10^{15}-10^{16} \mathrm{eV}\right)$ energy region where a bent is observed in primary cosmic ray all-particle energy spectrum suggesting their acceleration limit at supernova remnants (SNRs) in our Galaxy. We have also studied the cosmic ray anisotropy and the Sun's shadows in cosmic rays at high energies.

Meanwhile in the southern hemisphere, the HESS group reported on discovery of new 14 gamma-ray sources by galactic plane survey [W] in 2005. Surprisingly, most of them are UNIDentified (UNID) sources and faint in X-rays or other wavelengths. As the HESS survey was limited within the galactic plane in the southern hemisphere due to its narrow field of view, the importance of a wide field-of-view unbiased survey is emphasized. Furthermore, many of the 14 sources have a harder energy spectrum (indices: -1.8 to -2.8 ) at $\mathrm{TeV}$ energies than the standard candle Crab (index;-2.6). Although, the energy spectra were mostly measured below approximately $10 \mathrm{TeV}$ due to the sensitivity limit of the HESS air Cherenkov telescopes, they suggest that they extend up to the $100 \mathrm{TeV}$ region. The number of $\mathrm{TeV}$ sources now amount approximately to 200. However, the origin of the cosmic rays has not been clarified yet. Cosmic rays are supposed to be accelerated up to the knee energy (PeV) region at supernova remnants (SNRs) in our galaxy. Therefore, we naturally expect gamma rays at $100 \mathrm{TeV}$ energies, which originate in $\pi^{0}$ decays produced by the accelerated cosmic rays interacting with matter surrounding the SNRs. However, on-going experiments focus on measuring gamma rays in the $1 \mathrm{TeV}$ region. The gamma-ray emission of electron origin might be highly suppressed above $10 \mathrm{TeV}$ due to rapid decrease of inverse-Compton cross section by the Klein-Nishina effect as well as synchrotron radiation energy loss in the strong magnetic field around the SNRs. The detection and spectral measurement of gamma rays in the 100 $\mathrm{TeV}$ region from their celestial sources, together with multi-wavelength (radio, X-ray, gammaray) observations, will be a key experiment enabling us to discriminate between the two processes (cosmic-ray/electron origins), to locate the acceleration site (PeVatron which accelerate cosmic rays up to PeV energies) of cosmic rays and to verify the standard acceleration model of cosmic rays. The HESS 14 sources are very promising candidates for the cosmic-ray PeVatron. Furthermore, diffuse gamma rays from the Fermi bubbles recently reported by the Fermi-LAT group and sub-PeV neutrino events detected by IceCube suggests that the Fermi bubbles be a possible PeVatron candidate [D]. Similarly, the energy spectrum of diffuse gamma rays from the extended region around the galactic center marginally measured by HESS up to approximately $10 \mathrm{TeV}$ also strongly indicates existence of PeVatron [3], from which we expect to detect gamma rays at 100 $\mathrm{TeV}$ energies.

Under these circumstances, we built a large (approximately 4,000 $\mathrm{m}^{2}$ ) water Cherenkov-type muon detector array (MD) under the existing Tibet air shower array (AS) at 4,300 $\mathrm{m}$ above sea level, to observe 10-1000 TeV gamma rays from cosmic-ray accelerators in our Galaxy with wide 
field of view in the northern sky at very low background level. A gamma-ray induced air shower has significantly less muons compared with a cosmic-ray induced one. Therefore, we can effectively discriminate between primary gamma rays and cosmic-ray background events by means of counting number of muons in an air shower event by the muon detector array. We make a brief status report on the experiment.

\section{Tibet Air Shower Experiment}

The Tibet air shower array has been in operation at Yangbajing $\left(90^{\circ} 31^{\prime} \mathrm{E}, 30^{\circ} 06^{\prime} \mathrm{N} ; 4300 \mathrm{~m}\right.$ above sea level) in Tibet, China since 1990. It has continuously made a wide field-of-view (approximately 2 steradian) observation of cosmic rays and gamma rays in the northen sky.

The Tibet I array was constructed in 1990 [四], and it was gradually upgraded to the Tibet II by 1994 which consisted of 185 fast-timing (FT) scintillation counters placed on a $15 \mathrm{~m}$ square grid covering 36,900 $\mathrm{m}^{2}$, and 36 density (D) counters around the FT-counter array. Each counter has a plastic scintillator plate of $0.5 \mathrm{~m}^{2}$ in area and $3 \mathrm{~cm}$ in thickness. All the FT counters are equipped with a fast-timing 2-inch-diameter photomultiplier tube (FT-PMT), and 52 out of 185 FT counters are also equipped with a wide dynamic range 1.5-inch-diameter PMT (D-PMT) by which we measure up to 500 particles which saturates FT-PMT output, and all the D-counters have a DPMT. A $0.5 \mathrm{~cm}$ thick lead plate is put on the top of each counter in order to increase the counter sensitivity by converting gamma rays into electron-positron pairs in an electromagnetic shower. The mode energy of the triggered events in Tibet II is $\sim 10 \mathrm{TeV} / \sim 7 \mathrm{TeV}$ for cosmic rays/gamma rays [1], 目].

In 1996, we added 77 FT counters with a $7.5 \mathrm{~m}$ lattice interval to a $5,200 \mathrm{~m}^{2}$ area inside the northern part of the Tibet II array. We called this high-density array Tibet HD [ $[$ ]. The mode energy of the triggered events in Tibet $\mathrm{HD}$ is $\sim 3 \mathrm{TeV} / \sim 2 \mathrm{TeV}$ for cosmic rays/gamma rays. In the late fall of 1999, the array was further upgraded by adding 235 FT-counters to increase the high-density area from $5,200 \mathrm{~m}^{2}$ to $22,050 \mathrm{~m}^{2}$. We call this array and further upgraded one Tibet III [ [8]. In 2002, all of the $36,900 \mathrm{~m}^{2}$ area was covered by the high-density array by adding 200 FT-counters. Finally we set up 56 FT-counters around the $36,900 \mathrm{~m}^{2}$ high density array and equipped 8 D-counters with FT-PMT in 2003. At present, the Tibet air shower array consists of 761 FT-counters (249 of which have a D-PMT) and 28 D-counters [Q].

The performance of the Tibet air shower array has been well examined by observing the Moon's shadow (approximately 0.5 degree arc in diameter) in cosmic rays [ [ 8 , 口] . The def icit map of cosmic rays around the Moon demonstrates the angular resolution to be around $0.9^{\circ}$ at a few $\mathrm{TeV}$ for the Tibet III array. The pointing error is estimated to be less than $\sim 0.01^{\circ}$ by displacement of the shadow's center from the apparent center in the north-south direction, as the east-west component of the geomagnetic field is very small at the experimental site. On the other hand, the shadow center displacement in the east-west direction due to the geomagnetic field enables us to spectroscopically estimate the energy scale uncertainty less than $\pm 12 \%$ [ए0].

The first celestial TeV gamma-ray signal from the Crab (the standard candle in gamma-ra $\mathrm{y}$ astronomy) was detected by an imaging air Cherenkov telescope (IACT) developed by the Whipple group in 1989 [प]]. The pioneering work opened a new energy window in astronomy. 
Ten years later in 1999, we succeeded in observing multi-TeV gamma-ray signal from the Crab Nebula at 5.5 $\sigma$ confidence level, using the Tibet HD array [ $[$ ] $]$. This was the first detection of multi$\mathrm{TeV}$ gamma-ray signal by a conventional air shower array. Subsequently, in 2000, we reported on detection of multi-TeV gamma rays successfully at 3.7 $\sigma$ level from Mrk501 which was in a highly flaring state during March 1997 and August 1997 [ []]. We also succeeded in observing multi-TeV gamma-ray flares at $5.1 \sigma$ level from Markarian 421 which was in a very active phase during the year 2000 and 2001 [8]. Multi-TeV gamma-ray signal was successfully detected from the Crab by the Tibet-III array as well [U]].

We searched for $\mathrm{TeV}$ steady point sources in the northern sky. No statistically significant point source is found except for well established Crab and Mrk421 [ए2].

Search for steady PeV gamma-ray emission from the Monogem ring region is done with the Tibet data taken from 1997 to 2004 [ㅍ]]. No evidence for statistically significant gamma-ray signal is found in the Monogem ring where the MAKET-ANI experiment recently claimed a positive detection of PeV high-energy cosmic radiation [144], although our flux sensitivity is approximately 10 times better than MAKET-ANI's.

The 2-dimensional anisotropy $(\sim \pm 0.2 \%)$ in the equatorial coordinates are obtained for the first time in the multi-TeV energy range [15]]. Furthermore, we discovered a new diffuse cosmicray anisotropy (approximately $0.2 \%$ level excess) in the Cygnus region [ए6], although we cannot judge currently whether it is caused by gamma rays and/or a local cosmic-ray anisotropy. Overlapping the large-scale anisotropy in the Cygnus region, a few spacially separated enhancements of smaller scale were observed. These small-scale excesses $\left(\sim 2^{\circ}\right)$ hint at the extended gamma-ray emission[ए]]. The two of the small-scale excesses were shortly established[ए]], [ए8] as extended gamma-ray sources by the Milagro experiment capable of hadron/gamma-ray discrimination. Recently, we found a positive statistical correlation between the Fermi bright Galactic sources and our $\mathrm{TeV}$ gamma-ray excesses[ए]].

We also searched for multi-TeV diffuse gamma rays from the galactic plane [ [6, [20]. As there was no significant signal, flux upper limits were obtained.

Meanwhile, the Milagro group recently claimed detection of $\mathrm{TeV}$ diffuse gamma-ray signal in the Cygnus region along the galactic plane [2]]. Currently, we can not draw any clear conclusion on the Milagro result, as the Tibet air shower array is unable to distinguish between cosmic gamma rays and hadrons in the multi-TeV energy region at present.

To positively observe gamma rays in the $100 \mathrm{TeV}$ region with much better sensitivity than Tibet III, we decided to add an underground muon detector (MD) array to the existing air shower array. Gamma-ray induced electromagnetic air showers are muon-poor, while cosmic-ray induced hadronic ones are accompanied by many muons. This enables us to separate gamma rays from cosmic rays.

To confirm the hadron rejection power and construction feasibility of the MD array, we constructed a prototype muon detector in 2007.

\section{Prototype Muon Detector}

In the late fall of 2007, we constructed a prototype water Cherenkov muon detector (approximately $100 \mathrm{~m}^{2}$ ) at $\sim 90 \mathrm{~m}$ away from the center of the existing Tibet airshower array. The muon 
detector is made from reinforced concrete and composed of two water pool cells located at $2.5 \mathrm{~m}$ under the ground. Each cell is filled up with water of $1.5 \mathrm{~m}$ in depth, $7.2 \mathrm{~m} \times 7.2 \mathrm{~m}$ in area, equipped with three $20^{\prime \prime} \phi$ downward facing PMTs (HAMAMATSU R3600). Among the 3 PMTs, one is covered by a black sheet with $\sim 1 \%$ light transmission to effectively reduce the PMT gain (wider dynamic range), although we do not use it in this paper. The timing and charge information for each PMT is recorded by a trigger generated from surface scintillation counter array. We started the data taking in December, 2007. As for the air shower MC simulation, we generate air shower events by means of the CORSIKA code assuming the cosmic-ray composition estimated mainly from direct observational data. Our MC simulation reproduces well the experimental data obtained by the prototype muon detector. With the prototype muon detector, we set the most stringent upper limit on gamma-ray flux above $140 \mathrm{TeV}$ from the Crab [22].

\section{Tibet MD}

Success in the prototype muon detector pushed us to build a large underground muon detectors under the existing air shower array. Original concept (air shower array with $83,000 \mathrm{~m}^{2}$ in area +muon detectors with $\sim 10,000 \mathrm{~m}^{2}$ ) was demonstrated in [23], 24, 25] relevant to gamma-ray astronomy above $10 \mathrm{TeV}$.

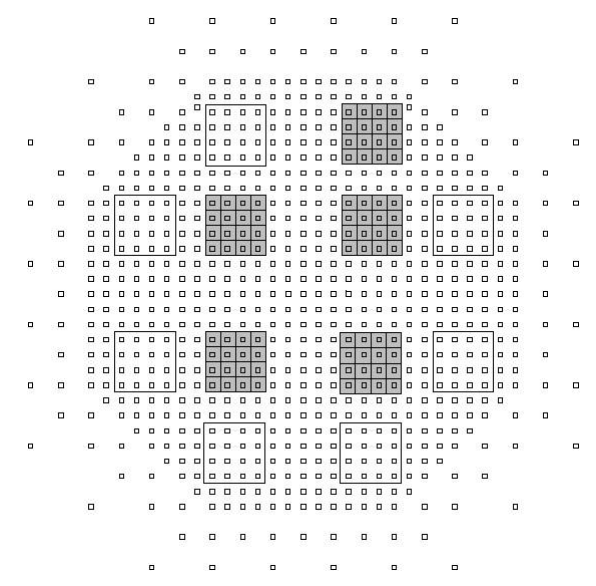

Figure 1: Schematic view of the Tibet MD. The grey five clusters were completed and started data-taking in 2014.

Realistically, we made up our mind to construct $\sim 4,000 \mathrm{~m}^{2}$ muon detectors as shown by the five big thick grey clusters in Fig. $\mathrm{W}$. Each muon detector cell is a waterproof concrete pool, $7.2 \mathrm{~m}$ wide $\times 7.2 \mathrm{~m}$ long $\times 1.5 \mathrm{~m}$ deep in size, equipped with two 20 inch-in-diameter photomultiplier tubes (PMTs), i.e., HAMAMATSU R3600, as shown in Fig. $\square$. The Tibet MD array is made up of five pool clusters ( 5 clusters $\times 16$ cells $=80$ muon detectors) set up $2.5 \mathrm{~m}$ underground. Its total effective area amounts approximately to $4,000 \mathrm{~m}^{2}$ for muons with energies more than $\sim 1 \mathrm{GeV}$.

Our current MC simulation predicts that the cosmic-ray background events will be rejected by approximately $99.9 \%$ at $100 \mathrm{TeV}$ using this $\mathrm{MD}$ array which will improve the sensitivity to gamma-ray sources by an order of magnitude around $100 \mathrm{TeV}$. The MD construction was completed and started data-taking in 2014. We are now accumulating data, while we are developing various analysis software tools to explore PeVatrons in the northern sky. 


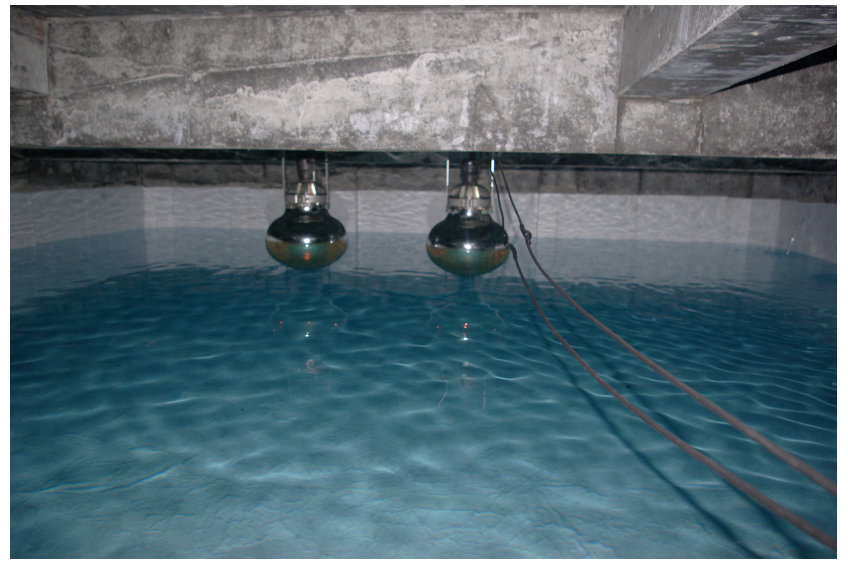

Figure 2: PMTs mounted on a MD cell filled with water

\section{Results and Discussions}

We estimate the expected integral sensitivity of the the Tibet AS+MD experiment to a pointlike gamma-ray source. Currently, the Tibet AS+MD experiment gives the world-best sensitivity ( $20 \% \mathrm{Crab}$ ) around $100 \mathrm{TeV}$ in the northern sky.

\section{Conclusions}

We built a large (approximately 4,000 $\mathrm{m}^{2}$ ) water Cherenkov-type muon detector array under the existing Tibet air shower array at 4,300 $\mathrm{m}$ above sea level, to observe $10-1000 \mathrm{TeV}$ gamma rays from cosmic-ray accelerators (PeVatrons) in our Galaxy with wide field of view in the northern sky at very low background level. The Tibet AS+MD started data-taking in 2014. We are currently accumulating data, while we are developing various analysis tools. The MD array will improve the sensitivity to gamma-ray sources by an order of magnitude around $100 \mathrm{TeV}$. The Tibet AS+MD experiment thus gives the world-best sensitivity around $100 \mathrm{TeV}$ in the northern hemisphere.

\section{Acknowledgments}

The collaborative experiment of the Tibet Air Shower Arrays has been performed under the auspices of the Ministry of Science and Technology of China (No. 2016YFE0125500) and the Ministry of Foreign Affairs of Japan. This work was supported in part by a Grant-in-Aid for Scientific Research on Priority Areas from the Ministry of Education, Culture, Sports, Science and Technology, by Grants-in-Aid for Science Research from the Japan Society for the Promotion of Science in Japan. This work is supported by the National Natural Science Foundation of China (Nos. 11533007 and 11673041) and the Chinese Academy of Sciences and the Key Laboratory of Particle Astrophysics, Institute of High Energy Physics, CAS. This work is supported by the joint research program of the Institute for Cosmic Ray Research (ICRR), The University of Tokyo.

\section{References}

[1] Aharonian, F.A. et al., Science 307, 1938 (2005). 
[2] Lunardini, C. et al., Phys. Rev. D 92, 021301 (2015).

[3] Abramowski, A. et al., Nature 531476 (2016).

[4] Amenomori, M. et al., Phys. Rev. Lett. 532, 2468 (1992).

[5] Amenomori, M. et al., Astrophys. J. 532, 302 (2000).

[6] Amenomori, M. et al., Astrophys. J. 580, 887 (2002).

[7] Amenomori, M. et al., Astrophy. J. 525, L93 (1999).

[8] Amenomori, M. et al., Astrophys. J. 598, 242 (2003).

[9] Amenomori, M. et al., Astrophys. J. 678, 1165 (2008).

[10] Amenomori, M. et al., Astrophys. J. 692, 61 (2009).

[11] Weeeks, T.C. et al., Astrophys. J. 342, 379 (1989).

[12] Amenomori, M. et al., Astrophys. J. 633, 1005 (2005).

[13] Amenomori, M. et al., Astrophys. J. 635, L53 (2005).

[14] Chilingarian, A. et al., Astrophys. J. 597, L129 (2003).

[15] Amenomori, M. et al., Astrophys. J. 626, L29 (2005).

[16] Amenomori, M. et al., Science 314, 439 (2006).

[17] Abdo, A.A. et al., Astrophys. J. 658, 776 (2007).

[18] Abdo, A.A. et al., Astrophys. J. 664, L91 (2007).

[19] Amenomori, M. et al., Astrophys. J. 709, L6 (2010).

[20] Amenomori, M. et al., Advances in Space Research 37, 1932 (2006).

[21] Atkins, R. et al., Phys. Rev. Lett. 95, 251103 (2005).

[22] Amenomori, M. et al., Astrophys. J. 813, 98 (2015).

[23] Amenomori, M. et al., AIP Conf. Proc. 1085, 723 (2008).

[24] Amenomori, M. et al., Journal of Physics: Conference Series 120, 062024 (2008).

[25] Sako, T.K. et al., Astropart. Phys. 32, 177 (2009). 